\title{
The Prevalence of General Dentists Who Screen for Obstructive Sleep Apnea
}

\author{
Harmeet K. Chiang, DDS, MS ${ }^{1}$; Alex Long, DDS²; Caroline K. Carrico, PhD¹; Riley Robinson, BS ${ }^{1}$ \\ ${ }^{1}$ School of Dentistry, Virginia Commonwealth University, Richmond, VA; ${ }^{2}$ General Dentist, Oceanside, CA
}

\begin{abstract}
Study Objectives: To determine the prevalence of general dentists screening for obstructive sleep apnea (OSA).
Methods: A brief, 12-item questionnaire consisting of a demographic section and questions regarding OSA screening methods, parameters, and preferences was sent to 1,000 general dentists across the United States using REDcap survey software.

Results: A total of 71 general dentists responded. Based on demographic results, it may be concluded that study participants represent a broad range of general dentists practicing in the United States. Participants were asked to select which screening modalities they use and to supply specifics regarding each modality to provide validity to responses. A total of $76 \%$ of participants reported screening for OSA. Most of them use more than one modality. Of participants responding affirmatively, $60 \%$ do not routinely screen more than $70 \%$ of patients. A total of $37 \%$ of participants ranked themselves a 3 or less (1-uncomfortable, 5-confident) regarding confidence in screening for OSA. There were $72 \%$ of participants who reported using a patient interview, $52 \%$ identified anatomical parameters, and $39 \%$ used patient questionnaires or tiredness surveys. Also, $41 \%$ of screening dentists have patients perform a home sleep test. There were $87 \%$ of participants suspecting OSA who referred patients to physicians for evaluation. Dentists with fewer than 30 years of experience were significantly more likely to screen for OSA than those with more than 30 years of experience ( $87 \%$ vs $63 \%, p=0.0459)$.
\end{abstract}

Conclusions: Most general dentists screen for OSA; however, most screening dentists lack confidence in performing accurate routine screenings and do so on fewer than $70 \%$ of patients.

Keywords: obstructive sleep apnea, OSA, general dentist, screening, prevalence

Citation: Chiang HK, Long A, Carrico CK, Robinson R. The prevalence of general dentists who screen for obstructive sleep apnea. $J$ Dent Sleep Med. 2018;5(3):55-60.

\section{INTRODUCTION}

Obstructive sleep apnea (OSA) is a condition caused by upper airway obstruction during sleep, characterized by recurrent breathing interferences, episodes of shallow breathing, and respiratory effort-related arousals, which can last for up to 30 seconds or longer. ${ }^{1,2}$ Immediate effects of the disrupted breathing cycle, associated with OSA, are usually associated with decreased blood oxygen levels. ${ }^{3}$ Among the most serious consequences of untreated OSA are highly increased risks of cardiovascular conditions such coronary artery disease, hypertension, congestive heart failure, and stroke. ${ }^{4}$ Furthermore, sleep deprivation is a common symptom reported by those with OSA, contributing to clinical depression, excessive daytime sleepiness, daytime fatigue accidents, and obesity. ${ }^{5-7}$

OSA is a common condition involving up to $24 \%$ of adult males and $9 \%$ of adult females; in many of these patients the condition goes undiagnosed for prolonged periods of time. ${ }^{8,9}$ It has been estimated that OSA remains undiagnosed in $82 \%$ to $93 \%$ of adults. ${ }^{10}$

Most Americans visit the dentist on a yearly basis. ${ }^{11}$ In order to provide greater awareness and improved outcomes of sleep apnea, a knowledge of general dentist involvement of OSA detection is imperative in aiding diagnosis, which may lead to multiple effective treatment options available to reduce negative effects associated with the disease. ${ }^{12}$ There are reports of the limited knowledge and training general dentists receive in detecting and treating OSA. ${ }^{13}$ However, there appears to be no data indicating the prevalence of dentists who screen for OSA. Therefore, this study is the first of its kind in representing the general dentist participation in screening for this serious condition.

\section{METHODS}

\section{Patients}

This study was approved as exempt by an Institutional Review Board (IRB: \#HM20003463). An email invitation was sent by a third-year dental student as a part of the research project in April 2015 to approximately 1,000 members of a national association of independent general dentists who were asked to participate in a survey using Research Electronic Data Capture (REDcap) survey software. The email invitation asked recipients if they had practiced dentistry within the past year and that participation in the survey would be greatly appreciated, but is completely voluntary and will remain anonymous. No compensation was provided for participation. A respondent was required to be a practicing general dentist to be eligible for inclusion in the analyses. 


\section{Questionnaire}

The questionnaire consisted of a demographic section (years in practice, dental school, practice specialty, practice setting, practice location) followed by a series of questions regarding screening methods, preferences, referrals, and treatment modalities. Three primary screening modalities were considered (medical history, sleep questionnaire, and anatomic parameters) as well as three additional modalities (cone beam computed tomography, home sleep test, twodimensional lateral cephalogram) and other modalities. The preferred modality was recorded, as was the percentage of patients routinely screened and the level of confidence in screening. Referral behavior and treatment services were recorded in the final portion of the questionnaire.

The primary outcome variable was whether any screening for OSA was reported. A positive answer or "Yes" was accepted if any of the primary or additional modalities were reported.

\section{Statistical Methods}

Responses were summarized using frequencies, percentages, and corresponding 95\% confidence intervals. The association between demographic factors and screening for OSA was determined first using univariate logistic regression. Overall association with OSA was assessed using multivariable logistic regression and post -hoc Tukey adjustment for multiple comparisons. A significance level of 0.05 and SAS EG v6.1 (SAS Institute, Inc., Cary, North Carolina, United States) was used for all analyses.

\section{RESULTS}

A total of 71 dentists responded to the questionnaire, resulting in a $7 \%$ response rate. Although this percentage is low, participants received training from more than 33 unique dental schools in the United States, practicing in 28 different states in various communities ranging from less than 20,000 people to more than 500,000 people (Table 1). There were $76 \%$ of dentists who reported screening for OSA. Participants were asked to select which screening modalities they utilize, and subsequently, specifics regarding each modality, aiming to provide some validity to their responses. A total of $61 \%$ reported using more than one screening modality (Table 2, Figure 1).

The patient interview is most frequently used $(72 \%$ of responders) and is the most preferred screening method, with almost all dentists identifying snoring (96\%) and daytime fatigue (84\%). Next, $51 \%$ reported identifying anatomical parameters, such as tonsils/adenoids (94\%) and neck circumference (92\%). Last, 39\% use a patient questionnaire or tiredness survey, with the Epworth Sleepiness Scale being most widely used $(86 \%)$, followed by the STOP-BANG questionnaire $(18 \%)$. It is important to note that $41 \%$ of screening dentists have patients undergo a home sleep test. Furthermore, $87 \%$ of dentists who identify a positive screening refer to a physician for further evaluation.

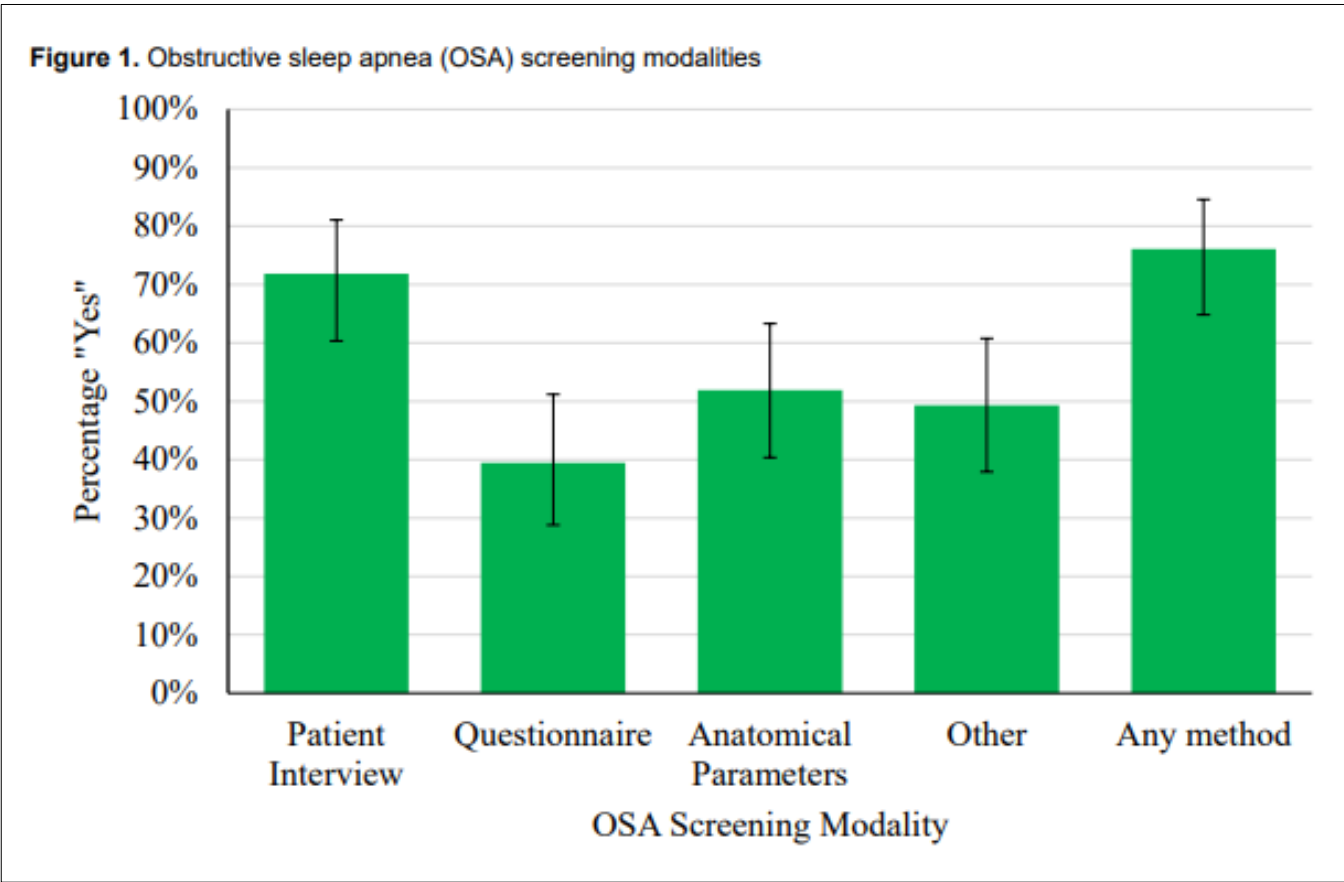


Table 1. Demographic characteristics of dentists $(n=71)$

\begin{tabular}{lcc} 
Demographic Factor & $\mathrm{N}$ & Percentages \\
\hline How many years have you practiced dentistry? & \\
Less than 5 & 4 & $5.6 \%$ \\
$5-10$ & 3 & $4.2 \%$ \\
$11-20$ & 6 & $8.5 \%$ \\
$21-30$ & 24 & $33.8 \%$ \\
More than 30 & 34 & $47.9 \%$ \\
In which setting are you primarily involved with dentistry? \\
Private Practice & 70 & $98.6 \%$ \\
Military & 1 & $1.4 \%$ \\
How would you describe your practice & setting? \\
Metropolitan (more than 50,000) & 18 & \\
Large City (50,000-50,000) & 21 & $36.5 \%$ \\
Small City (20,000-50,000) & 17 & $30.9 \%$ \\
Rural (less than 20,000) & 12 & $17.0 \%$ \\
Practice Location & \multicolumn{1}{l}{} \\
NC & 8 & $11.9 \%$ \\
NE & 21 & $31.3 \%$ \\
SE & 14 & $20.9 \%$ \\
SW & 10 & $14.9 \%$ \\
W & 14 & $20.9 \%$ \\
Dental School Location (DDS/DMD) & & \\
NC & 12 & $22.6 \%$ \\
NE & 16 & $30.2 \%$ \\
SE & 14 & $26.4 \%$ \\
SW & 7 & $13.2 \%$ \\
W & 7 & $13.2 \%$ \\
Other & 9 & $17.0 \%$ \\
\hline Nov & \\
\hline
\end{tabular}

Not every respondent replied to every question. The percentages were based on those responding to a given question. Practice and dental school location identified by collapsing the state into a grouping into districts. $\mathrm{NC}=$ North Central, NE $=$ North East, $\mathrm{SE}=$ South East, SW= South West, $\mathrm{W}=$ West.
Table 2. Prevalence of screening for obstructive sleep apnea (OSA)

Do you screen for OSA

N Percentages

\section{Primary Screening Modalities}

While obtaining medical history through a patient interview?

No $\quad 20 \quad 28.2 \%$

Yes

51

$71.8 \%$

Through a written sleep questionnaire?

No $\quad 43 \quad 60.6 \%$

Yes

28

$39.4 \%$

By specifically identifying anatomic parameters?

\begin{tabular}{lll} 
No & 34 & $48.6 \%$ \\
Yes & 36 & $51.4 \%$ \\
\hline
\end{tabular}

\section{Other Screening Modalities}

Cone beam computed tomography

$\begin{array}{lll}\text { No } & 61 & 85.9 \% \\ \text { Yes } & 10 & 14.1 \%\end{array}$

Home sleep test

$\begin{array}{lll}\text { No } & 42 & 59.2 \% \\ \text { Yes } & 29 & 40.8 \%\end{array}$

Two-dimensional lateral cephalogram

$\begin{array}{lrr}\text { No } & 70 & 98.6 \% \\ \text { Yes } & 1 & 1.4 \%\end{array}$

\section{Other methods}

\begin{tabular}{|c|c|c|c|}
\hline No & & 68 & $95.8 \%$ \\
\hline Yes & & 3 & $4.2 \%$ \\
\hline \multicolumn{4}{|c|}{ Any OSA screening } \\
\hline No & & 17 & $23.9 \%$ \\
\hline \multirow[t]{4}{*}{ Yes } & & 54 & $76.1 \%$ \\
\hline & 1 Modality & 11 & $15.5 \%$ \\
\hline & 2 Modalities & 9 & $12.7 \%$ \\
\hline & 3 or More Modalities & 34 & $47.9 \%$ \\
\hline
\end{tabular}

Figure 2. "Please characterize your confidence in screening for OSA." (1-uncomfortable, 5-confident)

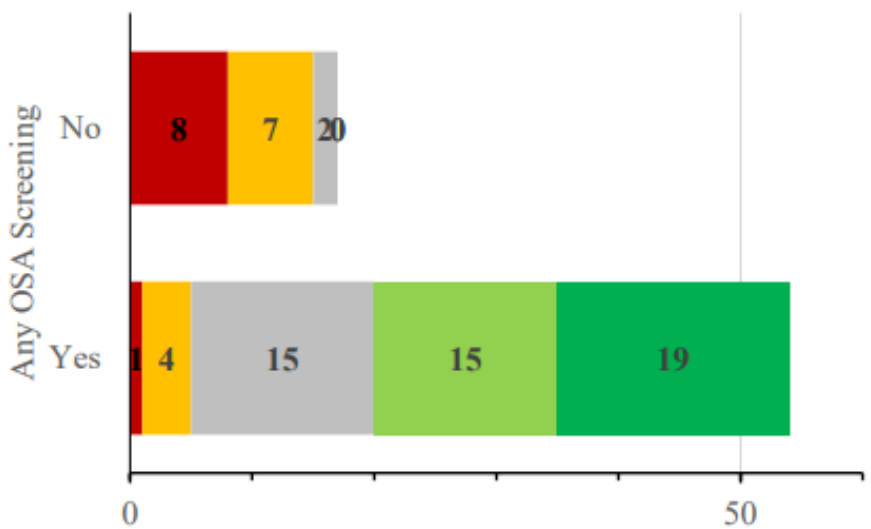

Table 3. "What percentage of patients do you routinely screen for OSA?"

\begin{tabular}{|l|l|}
\hline Dentist Response & Percentages \\
\hline$\leq 7$ out of 10 patients & $60 \%$ \\
\hline 8 out of 10 patients & $9 \%$ \\
\hline 9 out of 10 patients & $11 \%$ \\
\hline 10 out of 10 patients & $19 \%$ \\
\hline
\end{tabular}




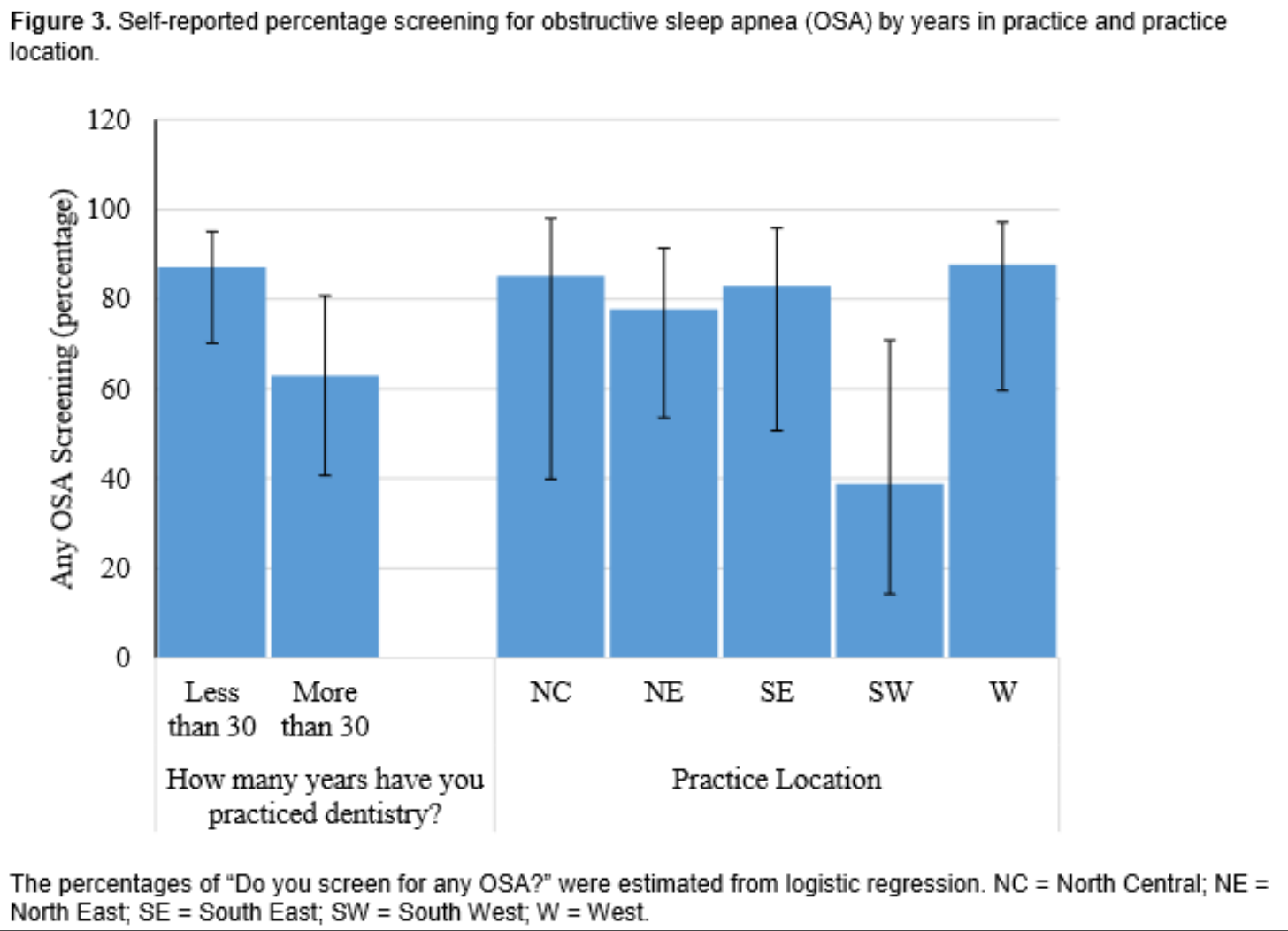

Sixty percent of dentists who screen for OSA are doing so for fewer than $70 \%$ of their patients (Table 3). Thirty-seven percent of responders reported themselves a 3 or lower on a confidence scale (1-uncomfortable, 5confident) in regard to screening for OSA (Figure 2).

In order to determine whether any demographic factor is associated with the prevalence of OSA screening, the chi-square test was used to test for an association with all of the factors described in Table 1. After collapsing years of practice into fewer than 30 years and 30 or more years (because of low number of practitioners with less experience), there was a significant association between years in practice and whether provider screening occurs $(\mathrm{p}$ $=0.0317)$. There was also marginal evidence of an association between screening for OSA and practice location $(p=0.0571)$. No other factors were significantly associated with screening for OSA $(p>0.5720)$. A multivariable logistic regression confirmed that there was a significant association between whether or not a provider reported screening for OSA and years of practice $(\mathrm{p}=$ 0.0459 ), but practice location was not statistically significant in the overall model ( $\mathrm{p}=0.1376$, Table 4 and Table 4. Relationships between demographic factors and screening for Obstructive Sleep Apnea (OSA)

Any OSA Screening

\begin{tabular}{lllll} 
Demographics & \multicolumn{3}{c}{ Percent } & \multicolumn{2}{c}{$95 \% \mathrm{Cl}$} \\
\hline \multicolumn{3}{l}{ How many years have you practiced dentistry? } \\
Less than 30 & 87.1 & a & $(70.2$ to & $95.1)$ \\
More than 30 & 62.9 & b & $(40.7$ to & $80.7)$ \\
& & & & \\
NC & 85.1 & a & $(39.9$ to & $98.0)$ \\
NE & 77.7 & a & $(53.5$ to & $91.4)$ \\
SE & 83.0 & a & $(50.7$ to & $95.9)$ \\
SW & 38.8 & a & $(14.2$ to & $70.8)$ \\
W & 87.6 & a & $(59.7$ to & $97.1)$ \\
\hline
\end{tabular}

Practice and dental school location identified by

collapsing the state into a grouping into districts. $\mathrm{Cl}=$ confidence interval, $\mathrm{NC}=$ North Central, NE $=$ North East, $\mathrm{SE}=$ South East, $\mathrm{SW}=$ South West, $\mathrm{W}=$ West.

Figure 3). Tukey post hoc test was used to identify the group differences and, compared to general dentists in practice more than 30 years, those in practice fewer than 30 years are significantly more likely to report screening for OSA $(87 \%$ versus $63 \%$, relative risk $=1.38)$. 


\section{DISCUSSION}

The parameters for diagnosing OSA are fairly straightforward. The gold standard is a polysomnography (PSG) performed at a sleep study center. This comprehensive test monitors heart rhythm, skeletal muscle activity, brain function, eye movement, pulse oximetry, and respiratory activity. ${ }^{14}$ In addition, home oximetry has become increasingly prevalent and is found to play an important role in screening for OSA. This test is easily performed in the comfort of a patient's own home but should only be used in conjunction with a PSG. ${ }^{14}$ One might discuss the role dentists play in screening patients for OSA, a role that is not well defined in the dental community.

A dentist's education and training regarding oropharyngeal anatomy and physiology is often more extensive than many practicing physicians. Not only are dentists trained to perform head, neck, and oral examinations, but currently the general population will often visit a general dentist more frequently than a physician. The prevalence of general dentists screening for OSA is generally unknown in the available literature. A plethora of research has been done in the dental field concerning treatment effectiveness and even diagnostic tools, but none can be found on screening prevalence and frequency. ${ }^{15}$

Dental screenings for OSA may be found using one tool, or a combination of three tools. First, a simple questionnaire that may be included in a health history form during new patient examinations or yearly health updates is used. The STOP-BANG questionnaire or Epworth Sleepiness Scale (ESS) are commonly used to gather information about factors such as tiredness, snoring, fatigue, blood pressure, and body mass index (BMI). ${ }^{16}$ Both questionnaires have proved to be effective screening tools by deeming patients low to high risk and in predicting airway obstruction. ${ }^{16}$ Although both questionnaires demonstrate significant positive association with OSA, it should be noted that the ESS has been shown to yield a weak significant correlation. ${ }^{17}$ Second, closely related to the questionnaire, is an interview between patient and doctor. Similar data, as previously stated, are collected and a risk assessment is made. Dentists may find more accurate information during a personal interview as opposed to a questionnaire, and therefore the interview may be a preferred method of screening. Last, a physical examination of head, neck, and oropharyngeal anatomy may be performed to evaluate specific structures and risk of OSA. This screening method is difficult to perform and is best used as an adjunct with patient-reported information. Dentists may assess BMI, neck circumference, mandible position, soft palate features, pharyngeal musculature, and Mallampati score in order to evaluate any signs of airway obstruction. ${ }^{18}$
This study reported a relatively low response rate for a REDcap survey of $7 \%$, in which $41 \%$ of the $76 \%$ of respondents who screen for OSA use a home sleep test. Because this ratio seems comparatively high in the overall general dentist population, self-selection bias may have occurred in this sample size.

Slightly more than $75 \%$ of general dentists screening for OSA seems like a very high percentage; however, the questions regarding screening confidence and percentage of patients screened may provide additional insights. From the data gathered, it appears there is a significant number of providers $(76 \%)$ who are performing routine screenings; however, of those who report screening, only $14 \%$ are screening every patient. Furthermore, only $45 \%$ of dentists are screening at least 7 of every 10 patients, resulting in most of the patient population not being screened. In addition, dentists report a lack of confidence in performing accurate routine screenings. Most of the responders (52\%) rated themselves a 3 or less (1-uncomfortable, 5-confident) on a confidence scale in screening for OSA. Therefore, most general dentists may report screening for OSA, but they are not screening every patient, and there is a lack of confidence in their ability to screen.

More dentists need to be educated and trained on proper and accurate screening methods for OSA so patients may receive proper care and treatment. With increased screening prevalence by general dentists more patients with this condition will be identified early and hopefully prevent complications and improve existing conditions. This study aims to raise awareness of the need for general dentists to routinely and accurately screen for OSA so patients with this condition may receive proper treatment and improved quality of life.

\section{ACKNOWLEDGMENTS}

Thanks to the Crown Council and Gregory Anderson for survey dissemination. Also, thanks to VCU School of Dentistry research department and the AD Williams Research Fellowship program, which provides support for dental students to participate in original and creative research and from award number UL1RR03190 from the National Center for Research Resources and NIH Roadmap for Medical Research, National Institutes of Health.

\section{ABBREVIATIONS}

BMI: Body Mass Index

ESS: Epworth Sleepiness Scale

IRB: Internal Review Board

OSA: Obstructive Sleep Apnea

PSG: Polysomnography

REDcap: Research Electronic Data Capture 


\section{REFERENCES}

1. Guilleminault C, Tilkian A, Adement WC. The sleep apnea syndromes. Annu Rev Med. 1976;27:465-484.

2. Padmanabhan V, Kavitha PR, Hegde AM. Sleep disordered breathing in children--a review and the role of a pediatric dentist. J Clin Pediatr Dent. 2010;35(1):15-21.

3. Brouillette RT, Fernbach SK, Hunt CE. Obstructive sleep apnea in infants and children. J Pediatr. 1982;100(1):31-40.

4. Punjabi NM. The epidemiology of adult obstructive sleep apnea. Am Thorac Soc. 2008; 5(2):136-143.

5. Bixler EO, Vgontzas AN, Lin HM, Calhoun SL, Vela-Bueno A, Kales A. Excessive daytime sleepiness in a general population sample: the role of sleep apnea, age, obesity, diabetes, and depression. J Clin Endocrinol Metab. 2005; 90(8):4510-4515.

6. Bishop TM, Ashrafioun L, Pigeon WR. The association between sleep apnea and suicidal thoughts and behavior: an analysis of national survey data. J Clin Psychiatry. 2018;79(1).

7. Ward KL, Hillman DR, James A, et al. Excessive daytime sleepiness increases the risk of motor vehicle crash in obstructive sleep apnea. $J$ Clin Sleep Med 2013;9(10):1013-1021.

Young T, Peppard PE, Gottlieb DJ. Epidemiology of obstructive sleep apnea: a population health perspective. Am J Respir Crit Care Med. 2002;165(9):1217-1239.

Finkel KJ, Searleman AC, Tymkew H, et al. Prevalence of undiagnosed obstructive sleep apnea among adult surgical patients in an academic medical center. Sleep Med. 2009; 10(7):7537-58.

10. Peppard PE, Young T, Barnet JH, Palta M, Hagen EW, Hla KM. Increased prevalence of sleep-disordered breathing in adults. Am J Epidemiol. 2013;177(9):1006-1014.

\section{SUBMISSION \& CORRESPONDENCE INFORMATION}

\section{Submitted for publication September 20, 2017 Submitted in final revised form February 22, 2018 Accepted for publication February 27, 2018}

Address correspondence to: Harmeet K. Chiang, DDS, MS; School of Dentistry, Virginia Commonwealth University $4^{\text {th }}$ Floor Lyons Building, 520 N. $12^{\text {th }}$ Street, Box 980566, Richmond, VA 23298, Email: hkchiang@vcu.edu
11. National Center for Health Statistics. Health, United States, 2016: With Chartbook on Long-term Trends in Health. Hyattsville, MD. 2017.

12. Jonas DE, Amick HR, Feltner C, et al. Screening for obstructive sleep apnea in adults: An evidence review for the U.S. preventative service task force. JAMA. 2017;317(4):415-433.

13. Bian H. Knowledge, opinions, and clinical experience of general dentists toward obstructive sleep apnea and oral appliances. Sleep Breath. 2004;8(2):85-90.

14. Epstein LJ, Kristo D, Strollo PJ, et al. Clinical guideline for the evaluation, management and long-term care of obstructive sleep apnea in adults. J Clin Sleep Med. 2009;5(3):263-276.

15. Sutherland K, Phillips CL, Cistulli PA. Efficacy versus effectiveness in the treatment of obstructive sleep apnea: CPAP and oral appliances. J Dent Sleep Med. 2015;2(4):175-181.

16. Silva GE, Vana KD, Goodwin JL, Sherrill DL, Quan SF Identification of patients with sleep disordered breathing: comparing the Four-Variable screening tool, STOP, STOP-Bang, and Epworth Sleepiness Scales. J Clin Sleep Med. 2011;7(5):467-472.

17. Heaton K, Anderson D. AA psychometric analysis of the Epworth sleepiness scale. J Nurs Meas. 2007;15(3):177-188.

18. Nuckton TJ, Gidden DV, Browner WS, Claman DM. Physical examination: Mallampati score as an independent predictor of obstructive sleep apnea. Sleep. 2006;29(7):903-908.

\section{DISCLOSURE STATEMENT}

Study data were collected and managed using Research Electronic Data Capture (REDCap) electronic data capture tools hosted at Virginia Commonwealth University (Harris et al. Research electronic data capture (REDCap) - a metadata-driven methodology and workflow process for providing translational research informatics support. J Biomed Inform. 2009;42(2):377-381.). REDCap is a secure, web-based application designed to support data capture for research studies. This was not an industrysupported study. The authors have indicated no financial conflicts of interest. 